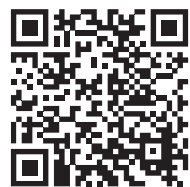

Octubre - Diciembre 2021 Vol. 1, núm. 1 / pp. 35-39

Palabras clave:

Ameloblastoma, articulación temporomandibular, prótesis maxilofacial, infecciones

relacionadas con prótesis, prótesis articulares.

Keywords: Ameloblastoma temporomandibular joint, joint prosthesis, prosthesis-related infections.

Citar como: Gatti PC, Florencia D, Ruiz

D, Giannunzio G.

Reconstrucción con prótesis customizada de articulación temporomandibular

tras resección de ameloblastoma. Lat Am J Oral Maxillofac

Surg. 2021; 1 (1): 35-39. https://dx.doi. org/10.35366/101916

* Cátedra de Cirugía y Traumatología Bucomaxilofacial I, Facultad de Odontología de la Universidad de Buenos Aires, Argentina.

₹ Servicio de Cirugía Maxilofacial del Hospital Zonal General de Agudos «General Manuel Belgrano».

Buenos Aires, Argentina.

Recibido: 16/08/2021

Aceptado: 29/08/2021

doi: 10.35366/101916

\title{
Reconstrucción con prótesis customizada de articulación temporomandibular tras resección de ameloblastoma
}

\author{
Customized temporomandibular joint prosthesis \\ reconstruction after ameloblastoma resection
}

\author{
Patricio César Gatti, ${ }^{\star}$ Diana Florencia, ${ }^{\ddagger}$ Damián Ruiz, ${ }^{\ddagger}$ Graciela Giannunzio ${ }^{\ddagger}$
}

\section{RESUMEN}

El ameloblastoma es un tumor odontogénico benigno localmente invasivo que tiene una alta tendencia a la recidiva. El tratamiento de elección en la mayoría de los casos es la resección con margen de seguridad. Cuando ésta es indicada debe tenerse en cuenta el tratamiento reconstructivo, que cuando incluye al cóndilo mandibular se debe realizar la reconstrucción de la articulación temporomandibular. En este trabajo se desarrolla un caso clínico de un paciente con diabetes tipo II y diagnóstico de ameloblastoma, al cual se le realizó la resección con margen de seguridad y en un segundo tiempo quirúrgico la reconstrucción con una prótesis personalizada de articulación temporomandibular (ATM). En este artículo se aborda el uso, indicaciones, prevención de complicaciones y resultados de la prótesis. La reconstrucción de la ATM a través de una prótesis personalizada es una opción segura y eficaz para el restablecimiento adecuado de la forma y la función del sistema estomatognático.
ABSTRACT

Ameloblastoma is a locally invasive benign odontogenic tumour that has a high tendency to recur. The first option for treatment in most cases is resection with a safety margin. When this is indicated, reconstructive treatment must be taken into account. When it involves the mandibular condyle, reconstruction of the temporomandibular joint must be performed. In this paper a clinical case of a patient with type II diabetes and a diagnosis of ameloblastoma is described. He underwent resection with a safety margin and in a second surgical stage reconstruction with a customized TMJ prosthesis was performed. The use, indications, prevention of complications and results of the prosthesis are discussed in this article. Reconstruction of the TMJ by means of a customized prosthesis is a safe and effective option for the adequate restoration of the form and function of the stomatognathic system.

\section{INTRODUCCIÓN}

El ameloblastoma es un tumor odontogénico benigno que representa $1 \%$ de todos los tumores y quistes de la región maxilofacial y $10 \%$ de los tumores odontogénicos. Clínicamente aparece como un tumor invasivo a nivel local, de crecimiento lento, por lo general asintomático y que ocasionalmente puede generar síntomas como inflamación, dolor, maloclusión y parestesia del lado afectado. ${ }^{1}$

Actual clasificación de tumores benignos de la OMS 2017:2

1. Ameloblastoma.

2. Ameloblastoma periférico o extraóseo.
3. Ameloblastoma uniquístico.

4. Ameloblastoma metastizante.

En cada caso la planificación del tratamiento quirúrgico dependerá de sus características histopatológicas, ubicación anatómica y extensión de la lesión, edad y estado general del paciente. ${ }^{3}$

El tratamiento de elección en la mayoría de los casos es la resección con margen de seguridad. Cuando ésta es indicada debe tenerse en cuenta el tratamiento reconstructivo que puede incluir injertos óseos o aloplásticos, colgajos libres, distracción osteogénica, etcétera. ${ }^{4}$

En casos en los que la lesión abarca al cóndilo mandibular puede realizarse la reconstrucción de la articulación temporomandibular (ATM) mediante una prótesis personalizada 


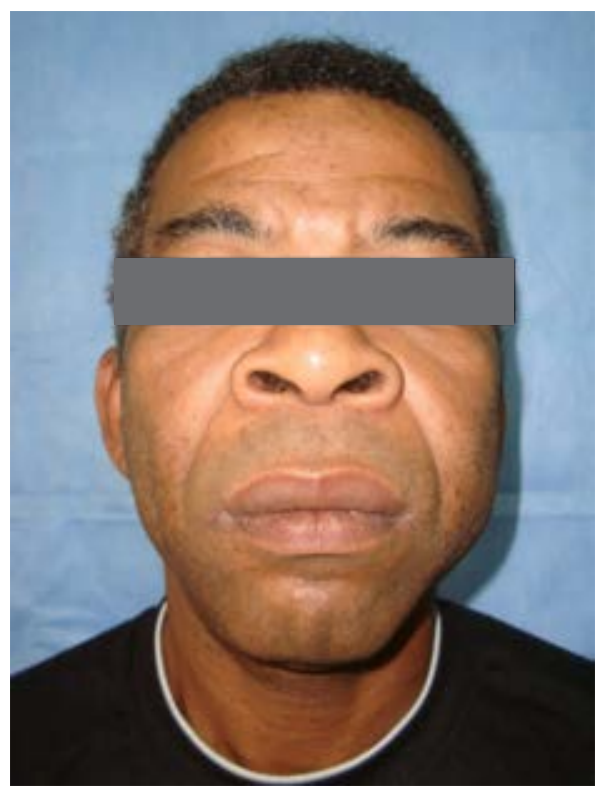

Figura 1: Preoperatorio.
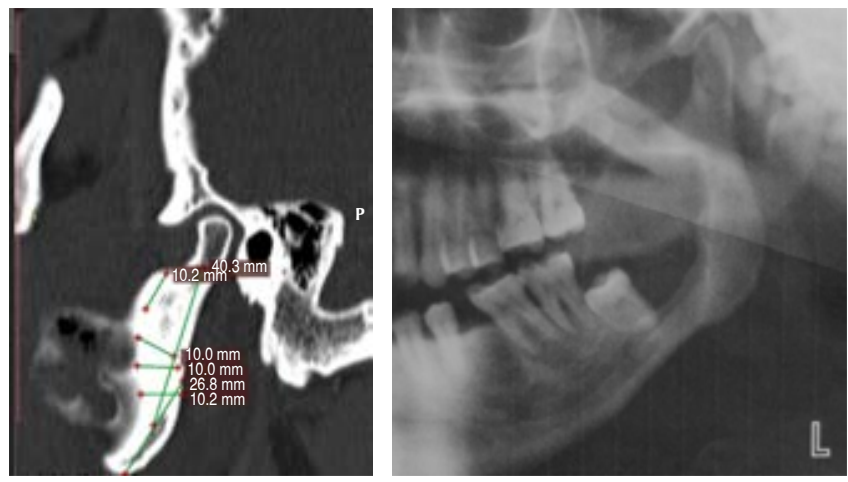

Figura 2: Imágenes preoperatorias.

para alcanzar los objetivos de restituir la forma, función, estabilidad y estética. ${ }^{5}$

El objetivo de este trabajo es presentar un caso clínico de un paciente con diabetes tipo II y diagnóstico de ameloblastoma multiquístico en maxilar inferior, quien luego de recibir el tratamiento resectivo intercurrió con una osteomielitis del cabo proximal (cóndilo mandibular); y por último se realizó la reconstrucción mediante una prótesis de ATM personalizada (Biomet/Lorenz).

\section{Caso clínico}

En enero de 2014 un paciente de 50 años de edad concurre al Servicio de Cirugía Maxilofacial del Hospital Zonal General de Agudos Manuel Belgrano presentando asimetría facial a causa de aumento de volumen en región geniana del lado izquierdo (Figura 1) con un estudio anatomopatológico con resultado de ameloblastoma multiquístico folicular en maxilar inferior. Como antecedente médico relevante el paciente padece diabetes tipo II. Se hicieron los estudios por imágenes de rutina (Figura 2) y en mayo de 2014, luego de firmar el consentimiento informado, se le realizó la resección segmentaria mandibular con un margen de seguridad de $1.5 \mathrm{~cm}$, el cual permitió conservar el cóndilo mandibular, y se efectuó la reconstrucción inmediata con una placa de reconstrucción TMS 2.4 (WL, BIOMET) (Figura 3).

A los 30 días postoperatorios el paciente evolucionó con una fístula orocutánea con secreción purulenta (Figura 4). En conjunto con el servicio de infectología y luego de no haber una respuesta favorable al tratamiento no invasivo se decidió el retiro de la placa de osteosíntesis y cóndilo mandibular.

En agosto de 2015 se planificó la reconstrucción simultánea del componente mandibular y articular (Figura 5). A partir de este

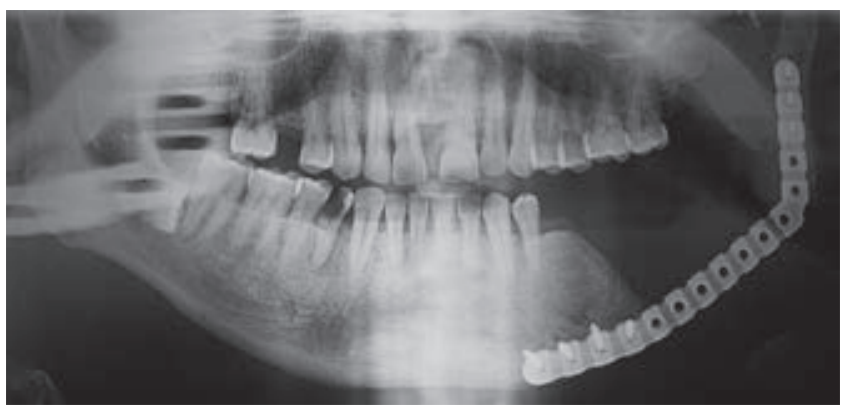

Figura 3: Radiografía post-resección.

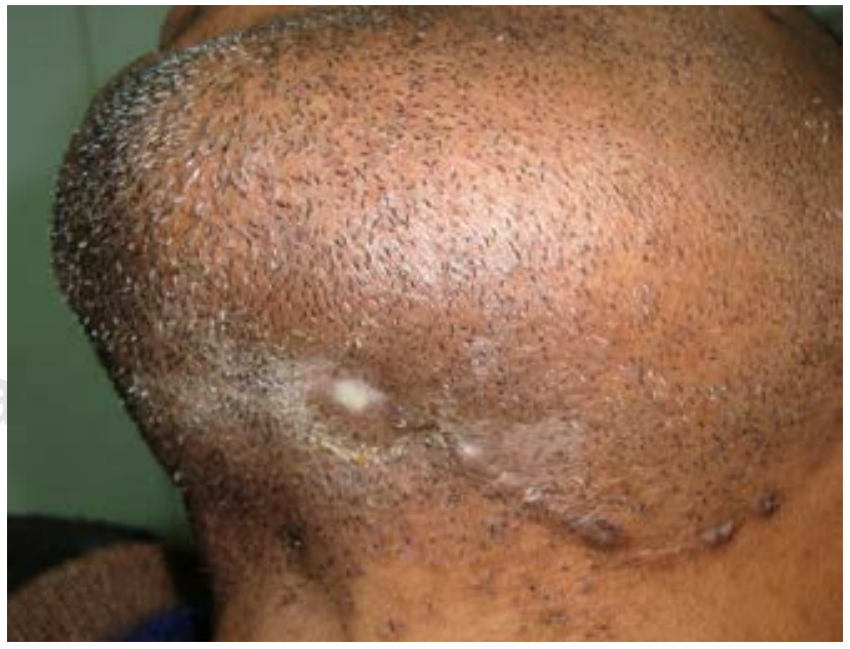

Figura 4: Fístula orocutánea. 

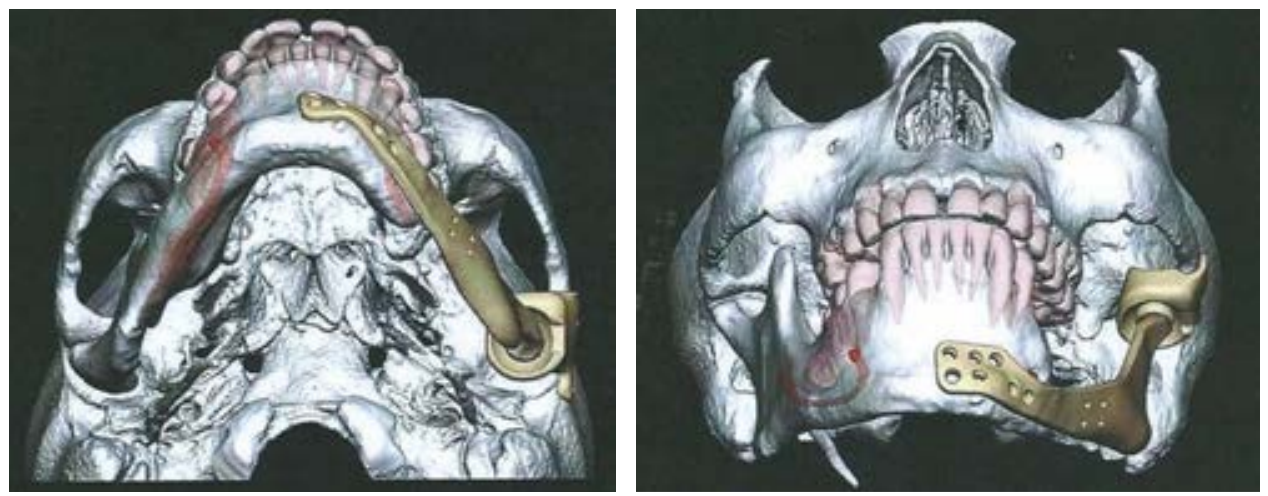

Figura 5:

Planificación digital de reconstrucción.

diseño se confeccionó una prótesis aloplástica personalizada Lorenz-Biomet (Biomet Microfixation, Jacksonville, FL, USA).

En conjunto con el servicio de endocrinología e infectología se preparó sistémicamente al paciente y en octubre de 2015 se realizó la cirugía reconstructiva, en la cual mediante un abordaje submandibular se insertó el componente mandibular y condilar, el cual fue fijado al cabo distal mandibular (Figura 6). Se realizó un segundo abordaje preauricular modificado a través del cual se insertó el componente correspondiente a la fosa glenoidea (Figura 7). Se recubrió el componente articular con una membrana de plasma rico en fibrina.

El postoperatorio transcurrió sin intercurrencias y se hicieron controles anuales (Figura 8). En el último control realizado en marzo de 2020 el paciente se presentó sin dolor, con una apertura bucal máxima de $40 \mathrm{~mm}$, y buena dinámica mandibular con una laterodesviación hacia el lado izquierdo de $7 \mathrm{~mm}$. Se observó una correcta estética y simetría facial (Figuras 9 y 10).

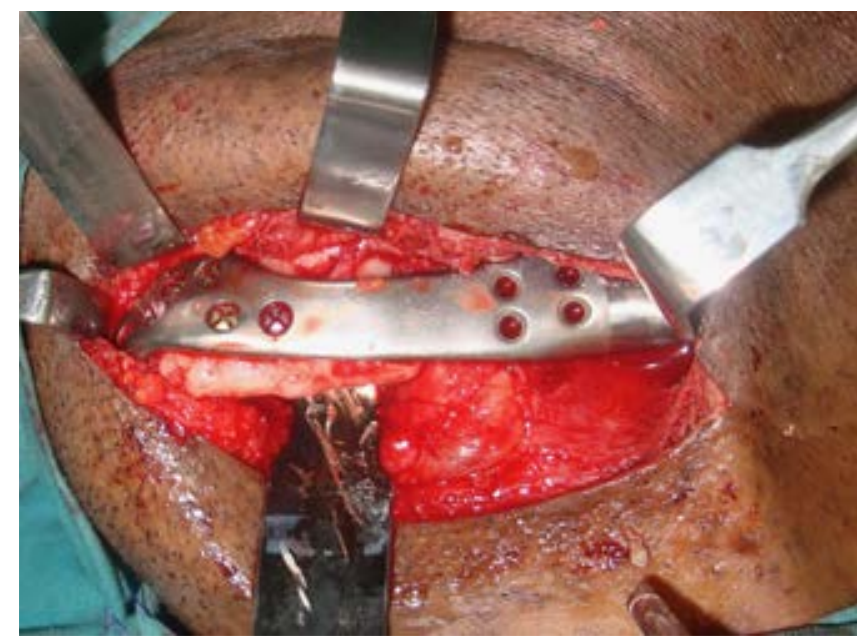

Figura 6: Abordaje submandibular.

\section{Discusıón}

La recurrencia del ameloblastoma está directamente relacionada con el tratamiento quirúrgico. Una revisión de 82 resecciones de ameloblastomas muestra que el tumor se extiende en un rango de 2 a $8 \mathrm{~mm}$ y en promedio $4.5 \mathrm{~mm}$ histológicamente más allá del límite radiográfico. Un margen de 1 a $1.5 \mathrm{~cm}$ provee un margen de tejido libre de patología, sin recurrencias en un periodo de cinco años. ${ }^{6}$

De los ameloblastomas, $60 \%$ se asienta en el ángulo y rama vertical de la mandíbula 3. Grafe en 1821 es quien describe por primera vez una hemimandibulectomía con desarticulación y desde esa época ha sido una terapéutica que se utiliza en diferentes situaciones clínicas, especialmente en el caso de tumores ubicados en esta zona. ${ }^{7}$ Esta terapéutica causa serios problemas funcionales y estéticos, es por eso que independientemente del método que se utilice para la reconstrucción los objetivos principales son restablecer la forma y la función. Se han descrito varias alternativas para la reconstrucción de estos defectos. ${ }^{1,4}$ Westermark y colaboradores reportan las ventajas

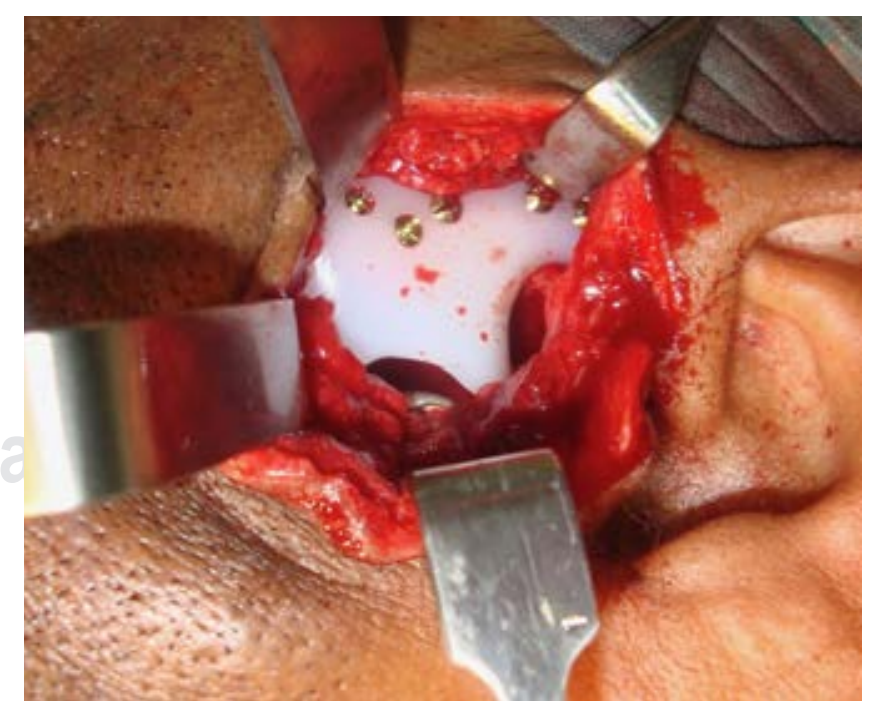

Figura 7: Abordaje preauricular. 


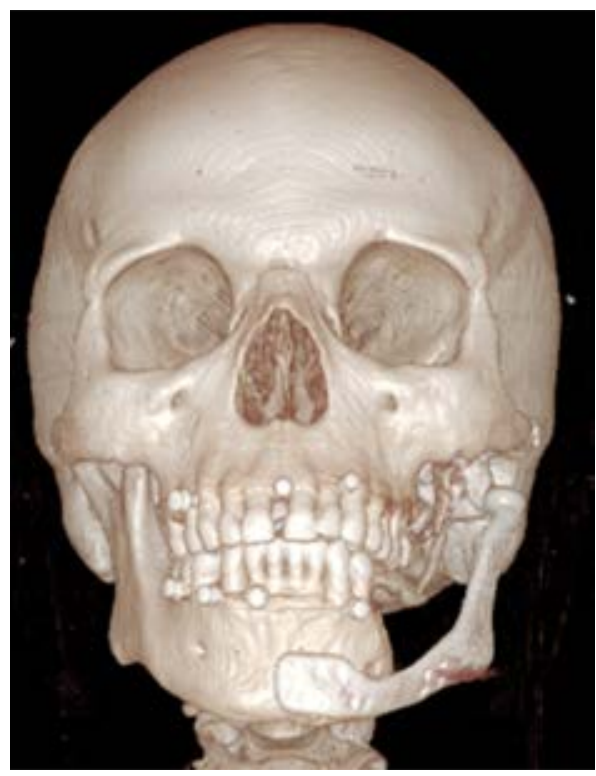

Figura 8: Tomografía post-reconstrucción inmediata.

en el uso de prótesis personalizadas en el caso de defectos amplios y complejos que involucran la ATM. ${ }^{8}$ A pesar de ser una técnica sensible y muy costosa, algunas de las ventajas sobre los injertos autógenos son que permite la función inmediata luego de la implantación y también elimina la necesidad de un sitio quirúrgico dador. ${ }^{5}$

En 1965 Christensen diseña la primera prótesis total de ATM (TMJ Inc., Golden, CO, USA)..$^{9}$ Entre 1981 y 1990 a miles de pacientes se les implantó la prótesis conocida en ese momento como Vitek-Kent (Vitek, Houston, TX, USA). La composición de los materiales resultó en el desprendimiento de partículas en la región de la fosa, reacciones a cuerpo extraño, destrucción de tejido blando y duro, y síndrome de dolor refractario incluso luego de retirar la prótesis. Esto llevó a la revocación de la aprobación de la FDA y trajo un descrédito en todos los reemplazos aloplásticos de ATM, lo cual conllevó al uso de procedimientos reconstructivos autógenos. ${ }^{10}$ Desde el año 2000 se han introducido nuevas prótesis y placas de reconstrucción para reemplazos totales de ATM y esto ha incrementado la demanda de reemplazos aloplásticos de ATM. ${ }^{9}$ Dentro de éstas se encuentran las prótesis de Biomet-Lorenz (Biomet Microfixation, Jacksonville, FL, USA) que combina una fosa de polietileno de ultra alto peso molecular (UHMWPE) y un componente mandibular y condilar de Co-Cr-Mo, que en la porción mandibular que va a estar en contacto con el tejido óseo se encuentra recubierta con un espray de plasma de titanio para aumentar la oseointegración. ${ }^{11}$

El principio más importante en la cirugía de implantación de prótesis de ATM es la estabilidad de los componentes protésicos. Las prótesis personalizadas se logran mediante la fijación con tornillos y la perfecta adaptación de los componentes a la anatomía del paciente. Esto evita o reduce los micromovimientos, los cuales son responsables de fracasos a largo término. ${ }^{12}$ Wolford y colaboradores en un estudio comparativo entre prótesis de stock y personalizadas concluyen que los pacientes implantados con prótesis personalizadas tienen mejores resultados estadísticamente significativos, tanto objetivos como subjetivos, que los implantados con prótesis de stock. ${ }^{13}$

En el caso clínico presentado el paciente muestra una laterodesviación hacia el lado de la prótesis. Sabine y colaboradores le atribuyen este fenómeno a que el cóndilo de la prótesis presenta una limitada o nula translación, es decir, que sólo rota. ${ }^{14,15}$ Este cambio funcional se cree que está relacionado con la formación de tejido cicatricial alrededor de la prótesis y con la pérdida parcial o total del músculo pterigoideo externo. Para compensar esta limitación, el diseño de la fosa reposiciona el punto de rotación hacia caudal. ${ }^{14,16}$

El paciente reportado en este trabajo padece de diabetes tipo II. Los niveles altos de glucosa en sangre se consideran un factor de riesgo de infección del sitio quirúrgico y si bien no existe un consenso en cuanto a los valores, se han sugerido niveles menores de $180 \mathrm{mg} / \mathrm{dL}$ el día de la cirugía que deben ser monitoreados en los periodos perioperatorio y postoperatorio. ${ }^{17}$ También el uso de antibióticos profilácticos intravenosos reduce el riesgo de infecciones postoperatorias. El conducto auditivo externo debe encontrarse libre de patología y ser ocluido durante la cirugía para prevenir la contaminación de la herida. Destacamos el cuidado que debe tenerse de no invadir la celda parotídea, lo cual puede resultar en la salida de saliva contaminada que puede propiciar también la infec-

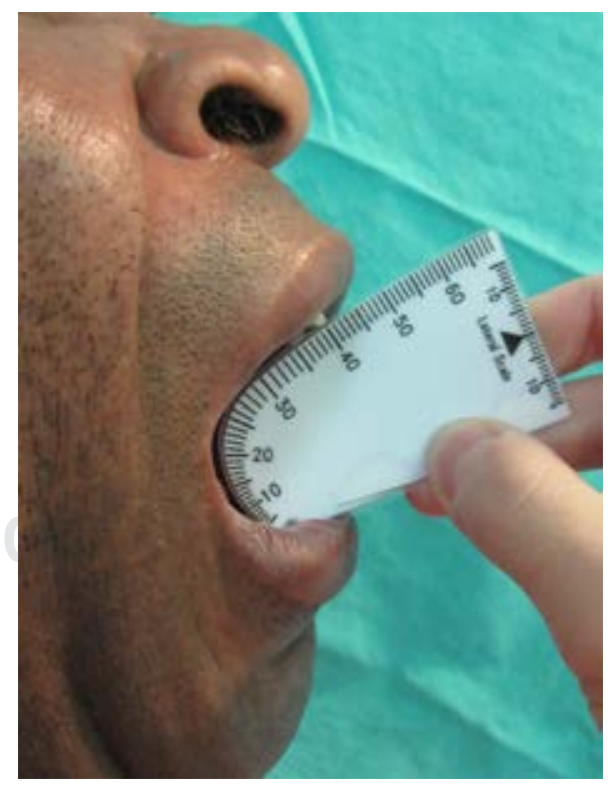

Figura 9: Apertura bucal último control. 


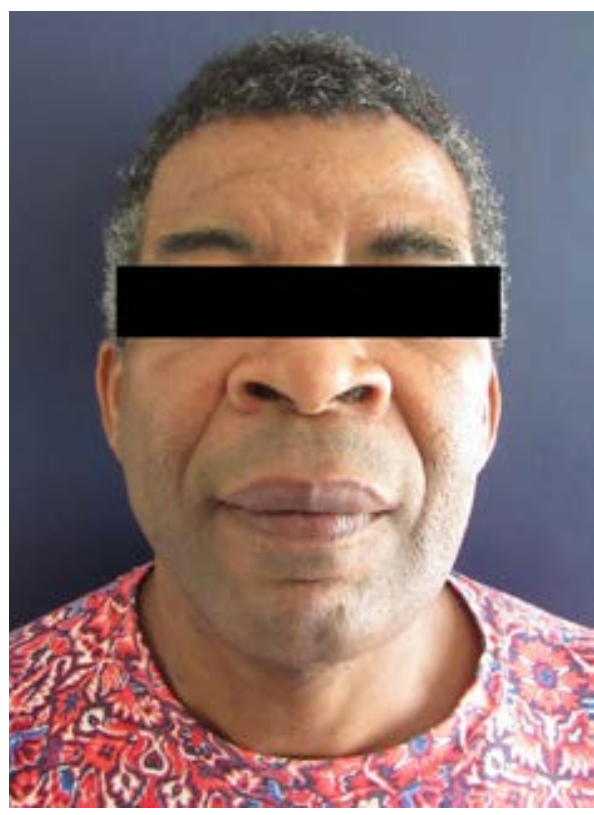

Figura 10: Postoperatorio último control.

ción. Asimismo, el tiempo de cirugía es un determinante de la infección del sitio quirúrgico y el uso de prótesis personalizadas lo disminuye considerablemente. ${ }^{18,19}$

La reconstrucción de la ATM a través de la instalación de una prótesis personalizada es una opción segura y eficaz para el restablecimiento adecuado de la forma y la función del sistema estomatognático. Su diseño, los materiales de fabricación y su estabilidad brindan resultados exitosos a largo plazo. La meticulosa planificación y preparación del paciente son indispensables para lograr el éxito.

\section{Referencias}

1. Bianchi B, Ferrari S, Copelli C, Sesenna E. Mandibular resection and reconstruction in the management of extensive ameloblastoma. J Oral Maxillofac Surg. 2013; 71: 528-537.

2. El-Naggar AK, Chan JKC, Grandis JR, Takata T, Slootweg PJ. World Health Organization classification of head and neck tumours. Volume 9. 4th ed. Lyon: IARC; 2017.

3. Hong J, Yun PY, Chung IH, Myoung H, Suh JD, Seo BM, et al. Longterm follow up on recurrence of 305 ameloblastoma cases. Int. J. Oral maxillofac. Surg. 2007; 36: 283-288.

4. Parameters of care: clinical practice guidelines for Oral and Maxillofacial Surgery (AAOMS ParCare 2012). Reconstructive surgery. J Oral Maxillofac Surg. 2012; 70 (Suppl 3): e272-e309.
5. Ruiz Valero CA, Duran-Rodriguez G, Solano-Parra N, Castro-Nuñez J. Inmediate total temporomandibular joint replacement with TMJ Concepts prosthesis as an Alternative for ameloblastoma cases. J Oral Maxillofac Surg. 2014; 72: 646.e1-646.e12.

6. Carlson ER. Ameloblastoma. symposuim on odontogenic tumors, AAOMS 82nd Annual Meeting and Scientific sessions, San Francisco, CA: 2000

7. Kademani D, Keller E. Iliac crest grafting for mandibular reconstruction. Atlas Oral Maxillofac Surg Clin North Am. 2006; 14 (2): 161-170.

8. Westermark A, Haden P, Aagaard E, Cornelius CP. The use of TMJ concepts prostheses to reconstruct patients with major temporomandibular joint and mandibular defects. Int J oral Maxillofac Surg. 2011; 40: 487-497.

9. Van Loon JP, De Bont GM, Boering G. Evaluation of temporomandibular joint prostheses: review of the literature from 1946 to 1994 and implications for future prosthesis designs. J Oral Maxillofac Surg. 1995; 53: 984-996.

10. Spagnoli D, Kent JN. Multicenter evaluation of temporomandibular joint Protoplast-Teflon disk implant. Oral Surg Oral Med Oral Pathol. 1992; 74: 411-421.

11. Aagaard E, Thygesen T. A prospective, single-centre study on patient outcomes following temporomandibular joint replacement using a custom-made Biomet TMJ prosthesis. Int J Oral Maxillofac Surg. 2014; 43: 1229-1235.

12. Mercuri LG. Alloplastic temporomandibular joint replacement: rationale for the use of custom devices. Int J Oral Maxillofac Surg. 2012; 41 (9): 1033-1040.

13. Wolford LM, Dingwerth DJ, Talwar RM, Pitta MC. Comparison of 2 temporomandibular joint total joint prosthesis systems. J Oral Maxillofac Surg. 2003; 61 (6): 685-690; discussion 690.

14. Linsen SS, Reich RH, Teschke M. Mandibular kinematics in patients with alloplastic total temporomandibular joint replacement - A prospective study. J Oral Maxillofac Surg. 2012; 70: 2057-2064.

15. Shan Yong Z, Liu H, Yang C, Zhang X, Abdelrehem A, Zheng J, et al. Modified surgical techniques for total alloplastic temporomandibular joint replacement: One institution's experience. J Craniomaxillofac Surg. 2015; 43 (6): 934-939.

16. Ghanaati S, Booms P, Orlowska A, Kubesch A, Lorenz J, Rutkowski J, et al. Advanced platelet-rich fibrin: a new concept for cell-based tissue engineering by means of inflammatory cells. J Oral Implantol. 2014; 40 (6): 679-689.

17. Marchant MH Jr, Viens NA, Cook C, Vail TP, Bolognesi MP. The impact of glycemic control and diabetes mellitus on perioperative outcomes after total joint arthroplasty. J Bone Joint Surg Am. 2009; 91 (7): 1621-1629.

18. Mercuri LG. Avoiding and managing temporomandibular joint total joint replacement surgical site infections. J Oral Maxillofac Surg. 2012; 70: 2280-2289.

19. Mercuri LG, Psutka D. Perioperative, postoperative, and prophylactic use of antibiotics in alloplastic total temporomandibular joint replacement surgery: a survey and preliminary guidelines. J Oral Maxillofac Surg. 2011; 69 (8): 2106-2111.

\section{Correspondencia:}

Patricio César Gatti

E-mail: patricio.gatti@odontologia.uba.ar 